TITLE:

\title{
Quantum-theoretical origin of the Lorentz invariance of the elementary-particle theory
}

$\operatorname{AUTHOR}(S)$ :

Nakanishi, Noboru

CITATION:

Nakanishi, Noboru. Quantum-theoretical origin of the Lorentz invariance of the elementary-particle theory. International Journal of Modern Physics A 2014, 29(06): 1450034.

ISSUE DATE:

2014-02-25

URL:

http://hdl.handle.net/2433/199868

\section{RIGHT:}

Electronic version of an article published as Int. J. Mod. Phys. A, 29, 1450034 (2014) DOI: 10.1142/S0217751X14500341 (C) World Scientific Publishing Company.; この論文は出版社 版でありません。引用の際には出版社版をご確認ご利用ください。; This is not the published version. Please cite only the published version. 


\title{
QUANTUM-THEORETICAL ORIGIN OF THE LORENTZ INVARIANCE OF THE ELEMENTARY-PARTICLE THEORY
}

\author{
NOBORU NAKANISHI \\ Professor Emeritus, Research Institute for Mathematical Sciences, \\ Kyoto University, Kyoto 606-8502, Japan \\ nbr-nak@trio.plala.or.jp \\ Received Day Month Year \\ Revised Day Month Year
}

\begin{abstract}
The reason why the elementary-particle theory must be Lorentz invariant is investigated under the principle of quantum priority, which implies that the existence of any spacetime manifold such as the Minkowski space should not be assumed logically prior to the formulation of quantum-theoretical framework. The consideration is based on the canonical operator formalism of quantum Einstein gravity, which is formulated without introducing any classical background spacetime nor any particular metric signature. The Lorentz invariance of the elementary-particle theory is derived as a consequence of the spontaneous breakdown of general linear invariance on the basis of quantum Einstein gravity. The pseudo-Riemannian spacetime can appear not as a consequence of the presence of gravitation but as that of the spontaneous breakdown of translational invariance.

Keywords: Lorentz invariance; quantum gravity; spontaneous breakdown.
\end{abstract}

PACS numbers:

\section{Introduction}

As is well known, in 1905, Einstein logically derived Lorentz invariance in any inertial frame on the basis of the principle of light-speed invariance and that of special relativity. Of course, his reasoning is completely classical; nevertheless, it was successfully applied to various quantum phenomena. Indeed, the brilliantly successful theory of elementary particles, the Standard Theory, was formulated under the hypothesis of Lorentz invariance. It has, however, never been seriously questioned why it must be Lorentz invariant. The purpose of the present paper is to answer this question.

It is natural to believe that the fundamental laws of Nature should be totally described by the quantum theory. On this belief, the present author ${ }^{1}$ previously proposed the following principle, called the "Principle of Quantum Priority": In the ultimate theory, any concept of classical physics must not appear logically prior to its quantum-theoretical construction. From this principle, the existence of any spacetime manifold such as the Minkowski space should not be assumed at the 
starting point of constructing the ultimate theory. That is, such a classical spacetime structure must be derived a posteriori.

At the beginning, we consider the (ordered) set of four real numbers, namely, $\mathbb{R}^{4}=\left\{\left(x^{0}, x^{1}, x^{2}, x^{3}\right) \mid x^{\mu} \in \mathbb{R},(\mu=0,1,2,3)\right\}$. We assume that the theory should be invariant under the translations and the $G L(4)$ transformations, that is, $\mathbb{R}^{4}$ is supposed to be a 4-dimensional affine space. ${ }^{\text {a }}$ This affine space is called "urspacetime". We emphasize that the ur-spacetime has no metric structure; we do not regard it as a manifold. ${ }^{\mathrm{b}}$

For later use, we need to introduce the concept of "ur-time". It is an arbitrary linear function of $x^{\mu}$. That is, let $c_{\mu}$ 's be any nontrivial four real constants and $a$ be any real constant; then the ur-time is $c_{\mu} x^{\mu}+a$. If the theory is affine invariant, it is independent of the choice of $c^{\mu}$ and $a$. Hence, for simplicity, we hereafter choose $c_{0}=1, c_{1}=c_{2}=c_{3}=0, a=0$, that is, $x^{0}$ is the ur-time.

Our primary purpose is to derive the Lorentz invariance of the elementaryparticle theory logically. As a matter of course, nothing can be derived if nothing is assumed. That is, we must assume some theoretical framework. We first state our standpoint in the following.

The theory of elementary particles ${ }^{c}$ is usually formulated in the Minkowski spacetime without including the gravity. But, of course, every elementary particle has gravitational interaction, and hence consistency requires the introduction of quantum gravity. ${ }^{\mathrm{d}}$ Since gravitational interaction is known to have close relationship with the spacetime structure, quantum gravity is more fundamental than the theory of elementary particles. Therefore, in order to discuss the spacetime structure, we should start with the quantum theory of gravitation.

Although various kinds of formulations have been proposed as the theories of quantum gravity, the BRS-formulated canonical operator formalism of the gravitational field ${ }^{2,3}$ is unique among them in the sense that the unitarity of its physical S-matrix is guaranteed at the same level of rigor as that of the gauge theories. This formalism is formulated manifestly $G L(4)$-covariant in the Heisenberg picture, that is, it is affine invariant so that the $x^{\mu}$ appearing in this theory can be regarded as the ur-spacetime.

\footnotetext{
a Note that the 4-dimensional affine tranformation is uniquely characterized as the bi-analytic mapping from $\mathbb{R}^{4}$ to $\mathbb{R}^{4}$.

${ }^{\mathrm{b}}$ The reason why we do not want to regard it as a manifold is as follows: A manifold is a geometric object and $x^{\mu}$ is introduced as a secondary quantity describing local coordinates. On the contrary, we want to regard $x^{\mu}$ as a primary quantity independent of a geometrical object. Partial differentiations with respect to $x^{\mu}$ should be understood as formal operations in the ur-spacetime; this is because we encounter products of quantum fields (operator-valued distributions) whose arguments are the same $x^{\mu}$.

${ }^{c}$ For convenience of description, the graviton is not treated as a kind of elementary particles.

dSome people wish to deny quantizing gravity and regard general relativity as an effective theory. But nobody has succeeded in constructing a consistent theory realizing such a standpoint. Since gravity is known to have quantum action on the particle, it is natural that its reaction is also of quantum nature.
} 
Unfortunately, there prevails the following opinion: "Since the covairant theory of quantum gravity is not renormalizable, it cannot be regarded as a physically sensible theory." This assertion is, however, wrong ${ }^{4}$ because nonrenormalizability is based on perturbation theory, which is wrong in quantum gravity. Indeed, in order to apply perturbation theory to quantum gravity, one must assume that the 0 -th order approximation of the quantum gravitational field is a classical spacetime metric tensor, but the correct 0 -th order approximation is not a c-number but a q-number.

In $\S 2$, we very briefly review the BRS-formulated canonical operator formalism of quantum Einstein gravity with making slight reformulation so as to be more appropriate to the discussion of the present paper. In $\S 3$, we show that the theory of elementary particles becomes Lorentz invariant as a result of the inevitable spontaneous breakdown of the $G L(4)$ invariance under the assumption that the translational invariance is not spontaneously broken. In $\S 4$, the case of spontaneously broken translational invariance is briefly discussed. In the final section, we summarize our reasoning and make some remarks.

\section{Quantum Einstein gravity}

As is well known, the classical Einstein gravity, namely, the theory of general relativity, is formulated on the basis of the equivalence principle and of the principle of general relativity. The theory is manifestly covariant under the general-coordinate transformations and the gravitational field $g_{\mu \nu}(x)$ is identified with the spacetime metric tensor. It is important, however, to notice that the Einstein equation is independent of metric signature; the Lorentzian signature of the Riemannian manifold is introduced as a boundary condition afterwards.

When quantizing Einstein gravity, it is not an adequate idea to still identify $g_{\mu \nu}(x)$ with the metric tensor, because we do not know its a priori metric signature. $^{\mathrm{e}}$ What should be quantized is, of course, the gravitational field; the metric tensor is purely of a classical concept, and it should be given by the vacuum expectation value of the quantum gravitational field $g_{\mu \nu}(x)$ afterwards. Furthermore, it is also not an adequate idea to suppose that the quantum gravitational field consists of the classical metric tensor and of the quantum fluctuation around it; the $a$ priori existence of the classical metric tensor evidently contradicts the principle of quantum priority.

Although the Einstein equation is independent of metric signature, in order to write down the action integral, it is necessary to introduce the square root of $\operatorname{det} g_{\mu \nu}(x)$. Since we do not know the metric signature a priori, it is impossible to define it without contradicting the hermiticity of the action integral. ${ }^{\mathrm{f}}$ The only way-

\footnotetext{
e Note that the sum over manifolds, that is encountered in the path-integral formulation of quantum gravity, has the trouble in how to specify the metric signature.

${ }^{\mathrm{f}}$ It is impossible to define a positive-definite operator in the framework of indefinite-metric quantum field theory.
} 
out of this difficulty is to introduce the "vierbein" field $h_{\mu}^{a}(x)(a=0,1,2,3)$, with banning its geometrical interpretation, through

$$
g_{\mu \nu}(x)=\xi_{a b} h_{\mu}^{a}(x) h_{\nu}^{b}(x),
$$

where the matrix formed by $\xi_{a b}$ is symmetric (because $g_{\mu \nu}(x)$ is symmetric) and nonsingular (because $\operatorname{det} g_{\mu \nu}(x) \neq 0$ ). According to Sylvester's theorem, we can assume without loss of generality that it is a diagonal matrix whose each diagonal element is either +1 or -1 . While $g_{\mu \nu}(x)$ has 10 independent components, $h_{\mu}^{a}(x)$ has 16 independent components. The extra 6 degrees of freedom imply the existence of a 6-dimensional gauge symmetry, which we call the "internal symmetry". They must be made totally unphysical; this condition will be seen to be satisfied in the construction of the quantum Einstein gravity. By using $h(x) \equiv \operatorname{det}_{\mu}^{a}(x)$, it is now possible to have a hermitian "invariant" volume element $h(x) d^{4} x$.

Now, we present a brief summary of the manifestly-covariant canonical operator formalism of the quantum Einstein gravity based on the BRS invariance. ${ }^{2,3}$ Here, the BRS invariance is the quantum version of the local-translation (i.e., generalcoordinate transformation) invariance; since the arbitrary transformation function in the classical Einstein gravity is replaced by the local-translation FP ghost, any local-translationally invariant (in the classical sense) quantity is invariant under the BRS transformation of the quantum Einstein gravity.

The fundamental fields are the vierbein field $h_{\mu}^{a}(x)$, the local-translation B-field $b_{\rho}(x)$, the local-translation $\mathrm{FP}$ ghost $c^{\sigma}(x)$ and anti-ghost $\bar{c}_{\tau}(x)$, together with the internal-symmetry B-field, the internal-symmetry FP ghost and anti-ghost, and various fields of elementary particles. We consider elementary particles generically, that is, we do not work with any concrete model of elementary particles. The fundamental action $S$ is the integral of the Lagrangian density $\mathcal{L}(x)$ over $\mathbb{R}^{4}$. It consists of the Einstein-Hilbert action, the local-translation gauge-fixing term (given by the de Donder-condition formula multiplied by $b_{\rho}(x)$ ) plus FP-ghost one, the internalsymmetry gauge-fixingg plus FP-ghost one and the action, denoted by $S_{M}$, of the fields of elementary particles. Of course, each term of $S$ is invariant under (global) translations, $G L(4)$ transformations, two kinds (local translation and internal gauge symmetry) of the BRS transformations. ${ }^{\text {h }}$

The field equations are derived from the variational principle $\delta S=0$ as usual. ${ }^{i}$ In order to define the operator properties of quantum fields, the canonical quantization procedure is performed with respect to the ur-time $x^{0}$. The canonical fields are all fields presented above except for $b_{\rho}(x)$ (together with some possible multiplier fields). The canonical conjugate to each canonical field is defined as usual and the

\footnotetext{
g This gauge-fixing term must be local-translationally invariant.


which is denoted by $\operatorname{IOSP}(8,8)$, containing 144 independent generators, ${ }^{5}$ together with the internal symmetry containing 29 independent generators.

iThe infinitesimal variation of each quantum field is a c-number function.
} 
canonical commutation or anti-commutation relations are set up at the equal urtimes. The choice of commutation or anti-commutation must be consistent with the BRS invariance of $S$, and to choose anti-commutation is admitted only if $S$ remains invariant under the simultaneous sign change of both relevant quantities. The usual spin-statistics relation is outside of our consideration, because all fields of elementary particles are scalar fields with respect to the ur-spacetime. ${ }^{\mathrm{j}}$ The Dirac or Weyl field is a spinor and the gauge field is a vector with respect to the internal symmetry. ${ }^{\mathrm{k}}$ Hereafter, for simplicity, we omit stating the prefix "anti" for anti-commutation explicitly.

The equal ur-time commutation relations concerning the canoninal fields and their first-order ur-time derivatives can be explicitly calculated from the canonical commutation relations; especially, it is quite remarkable that all equal ur-time commutation relations concerning $h_{\mu}^{a}(x), b_{\rho}(x)$, etc. and their first-order derivatives are obtained in closed form. ${ }^{7}$

In general, the method for solving quantum field theory in the Heisenberg picture $^{8}$ is, in principle, as follows. First, taking the 4-dimensional commutators between the left-hand side of each field equation and each field, we rewrite all field equations into the equations for these 4-dimensional commutators. Then, regarding the equal-time commutation relations as initial conditions, we set up a q-number Cauchy problem for the set of the 4-dimensional commutators. If we can solve it, we obtain the operator algebra of the fields (a kind of an infinite-dimensional Lie algebra); all multiple commutators are calculable at least in principle.

A representation of this operator algebra is constructed, so as to be consistent with all the above multiple commutators, in terms of a set of Wightman functions $\left\langle 0\left|\Phi_{1}\left(x_{1}\right) \cdots \Phi_{n}\left(x_{n}\right)\right| 0\right\rangle$, where $\Phi_{k}\left(x_{k}\right)$ is a field operator and $|0\rangle$ denotes the vacuum state. It is generally impossible to require the norm positivity of state vectors, that is, the representation space is usually an indefinite-metric Hilbert-like space. As a property of the vacuum state, we require the following energy-positivity condition: ${ }^{8}$ Any $n$-point truncated ${ }^{1}$ Wightman function be a distribution defined as a boundary value of an analytic function of $x_{1}^{0}-x_{2}^{0}, \cdots, x_{n-1}{ }^{0}-x_{n}^{0}$ holomorphic (and asymptotically vanishing) in the lower-half planes. ${ }^{\mathrm{m}}$

If the theory under consideration is BRS invariant, we must introduce the corresponding Kugo-Ojima subsidiary condition $Q_{B} \mid$ phys $\rangle=0,{ }^{n}$ where $Q_{B}$ is the BRS charge and $\mid$ phys $\rangle$ denotes a physical state. If the physical subspace, defined by

\footnotetext{
jIt should be noted that there is no spinor representation of $G L(4)$.

${ }^{\mathrm{k}}$ For a gauge field $A_{\mu}(x)$, we adopt $A_{a}(x)=h_{a}^{\mu}(x) A_{\mu}(x)$ as the canonical field. ${ }^{6}$

${ }^{1}$ The word "truncated" means to subtract the contributions from vacuum intermediate states.

m The background of the energy-positivity condition is the fact that the Fourier representation of the truncated Wightman function with respect to those $n-1$ variables contains the factor $\exp (-i)\left[E_{1}\left(x_{1}^{0}-x_{2}^{0}\right)+\cdots+E_{n-1}\left(x_{n-1}{ }^{0}-x_{n}^{0}\right)\right]$ in the integrand, where $E_{k}$ denotes the integration variable corresponding to the intermediate-state energy. If the Fourier transform vanishes unless $E_{k}>0$ for $k=1, \cdots, n-1$, the truncated Wightman function has the properties stated here. ${ }^{\mathrm{n}}$ In terms of Wightman functions, this is defined by $\left\langle 0\left|\Phi_{1}\left(x_{1}\right) \cdots \Phi_{n}\left(x_{n}\right) Q_{B}\right|\right.$ phys $\rangle=0$ for any set of $\Phi_{1}\left(x_{1}\right), \cdots, \Phi_{n}\left(x_{n}\right)$.
} 
the totality of the physical states, has a positive-semidefinite norm, the physical S-matrix is shown to be unitary.

The above procedure works well for some simple models. For more realistic theories, we need to expand everything in powers of a parameter involved in it; for the BRS-invariant theory, this expansion is different from the conventional perturbation theory, because the BRS invariance is kept in each order in our approach. ${ }^{8}$

We can apply the above procedure to the quantum Einstein gravity by regarding $x^{\mu}$ as ur-spacetime. Expanding it in powers of the Einstein gravitational constant $\kappa$, we can explicitly calculate the 0 -th order solution. We find that

$$
\left[g_{\mu \nu}{ }^{(0)}(x), g_{\lambda \rho}{ }^{(0)}(y)\right]=0
$$

but $\left[g_{\mu \nu}{ }^{(0)}(x), b_{\rho}{ }^{(0)}(y)\right] \neq 0$ and $\left[g_{\mu \nu}{ }^{(0)}(x), g_{\lambda \rho}{ }^{(1)}(y)\right] \neq 0$, where a superscript $(k)$ stands for the $k$-th order approximation. Thus, indeed, $g_{\mu \nu}{ }^{(0)}(x)$ is a q-number.

\section{Lorentz invariance of the elementary-particle theory}

In this section, we show how the Lorentz invariance of the elementary-particle theory is derived from the canonical operator formalism of the quantum Einstein gravity.

According to Noether's theorem, there are the (ur-spacetime) translation generators $P_{\mu}$, the (ur-spacetime) $G L(4)$ generators $\hat{M}_{\nu}^{\mu}$, and the (global) internalsymmetry generators $M^{a b}\left(=-M^{b a}\right)$. We write an elementary-particle field as $\varphi_{A}(x)$ generically. Then we have the following commutation relations: ${ }^{\circ}$

$$
\begin{gathered}
i\left[P_{\mu}, h_{\lambda}^{c}(x)\right]=\partial_{\mu} h_{\lambda}^{c}(x), \\
i\left[P_{\mu}, \varphi_{A}(x)\right]=\partial_{\mu} \varphi_{A}(x) ; \\
i\left[\hat{M}^{\mu}{ }_{\nu}, h_{\lambda}^{c}(x)\right]=x^{\mu} \partial_{\nu} h_{\lambda}^{c}(x)+\delta_{\lambda}^{\mu} h_{\nu}^{c}(x), \\
i\left[\hat{M}^{\mu}{ }_{\nu}, \varphi_{A}(x)\right]=x^{\mu} \partial_{\nu} \varphi_{A}(x) \\
i\left[M^{a b}, h_{\lambda}^{c}(x)\right]=\xi^{a c} h_{\lambda}^{b}(x)-\xi^{b c} h_{\lambda}^{a}(x), \\
i\left[M^{a b}, \varphi_{A}(x)\right]=\left(\sigma_{A}{ }^{B}\right)^{a b} \varphi_{B}(x),
\end{gathered}
$$

where $\xi^{a b}=\xi_{a b}$ and $\left(\sigma_{A}{ }^{B}\right)^{a b}$ represents the transformation matrix of $\varphi_{A}(x)$ under global internal-symmetry transformations. Of course, all these commutation relations are the expected ones, but it should be noted that they can be confirmed by explicit calculations. ${ }^{9}$ Likewise, the commutators between generators are also the expected ones; especially, both $P_{\mu}$ and $\hat{M}^{\mu}{ }_{\nu}$ are commutative with $M^{a b}$.

We assume that the translational invariance is not spontaneously broken, that is, $P_{\mu}|0\rangle=0$. Then, the vacuum expectation value of the commutator between $P_{\mu}$ and any local quantity is independent of $x^{\mu}$. Indeed, from (3.1), we have

$$
\begin{aligned}
\left\langle 0\left|\partial_{\mu} h_{\lambda}^{c}(x)\right| 0\right\rangle & =0, \\
\left\langle 0\left|\partial_{\mu} \varphi_{A}(x)\right| 0\right\rangle & =0 .
\end{aligned}
$$

${ }^{\circ}$ Likewise for the ghost fields $b_{\rho}(x)$, etc. 
From (3.2) and (3.3), the vacuum expectation values of the commutators involving the vierbein field are

$$
\begin{aligned}
& i\left\langle 0\left|\left[\hat{M}^{\mu}{ }_{\nu}, h_{\lambda}^{c}(x)\right]\right| 0\right\rangle=\delta_{\lambda}^{\mu} u_{\nu}^{c}, \\
& i\left\langle 0\left|\left[M^{a b}, \quad h_{\lambda}^{c}(x)\right]\right| 0\right\rangle=\xi^{a c} u_{\lambda}^{b}-\xi^{b c} u_{\lambda}^{a},
\end{aligned}
$$

where $u_{\mu}^{a} \equiv\left\langle 0\left|h_{\mu}^{a}(x)\right| 0\right\rangle$ is a constant matrix. In writing the action $S$, we have, of course, assumed the existence of the inverse matrix of the vierbein field; hence the vacuum expectation value of the vierbein field is invertible at least at $\kappa=0$. It is natural, therefore, to suppose that it is so also for $\kappa \neq 0$, that is, we assume that $\operatorname{det} u_{\mu}{ }^{a} \neq 0$. Then, from (3.4), all components of $\hat{M}^{\mu}{ }_{\nu}$ and all independent components of $M^{a b}$ are seen to be spontaneously broken. The Nambu-Goldstone boson of the symmetric part of the former is nothing but the graviton, and therefore the exact masslessness of the graviton is predicted. ${ }^{\mathrm{p} 10}$

Hereafter, we employ $\alpha, \beta, \cdots$ for the scripts $(=0,1,2,3)$ of any quantity independent of $x^{\mu}$. Let the transposed inverse matrix of $u_{\gamma}{ }^{\alpha}$ be $v_{\beta}^{\gamma}$ (namely, $u_{\gamma}^{\alpha} v_{\beta}^{\gamma}=\delta_{\beta}^{\alpha}$ ). We consider the quantity

$$
\tilde{M}^{\alpha \beta} \equiv\left(\xi^{\beta \gamma} u_{\delta}^{\alpha} v_{\gamma}^{\varepsilon}-\xi^{\alpha \gamma} u_{\delta}^{\beta} v_{\gamma}^{\varepsilon}\right) \hat{M}_{\varepsilon}^{\delta}+M^{\alpha \beta} .
$$

Then (3.4) yields

$$
\left\langle 0\left|\left[\tilde{M}^{\alpha \beta}, h_{\lambda}^{c}(x)\right]\right| 0\right\rangle=0 .
$$

As for the vacuum expectation value of the commutator between $\tilde{M}^{\alpha \beta}$ and an elementary-particle field, (3.2) and (3.3) yield

$$
\left\langle 0\left|\left[\tilde{M}^{\alpha \beta}, \varphi_{A}(x)\right]\right| 0\right\rangle=-i\left(\sigma_{A}^{B}\right)^{\alpha \beta}\left\langle 0\left|\varphi_{B}(x)\right| 0\right\rangle .
$$

Except for the case $\sigma_{A}^{B}=0$, it is natural to assume $\left\langle 0\left|\varphi_{B}(x)\right| 0\right\rangle=0$ because it is outside of our consideration the possibility that the spontaneous breakdown of the internal symmetry is caused by an elementary-particle field. Thus $\tilde{M}^{\alpha \beta}$ 's are the generators of spontaneously unbroken symmetry. We set $\tilde{x}^{\alpha} \equiv u_{\mu}{ }^{\alpha} x^{\mu}$, and therefore $\tilde{\partial}^{\alpha} \equiv \xi^{\alpha \beta} v^{\mu}{ }_{\beta} \partial_{\mu}$; then from (3.5) and (3.2), we have

$$
i\left[\tilde{M}^{\alpha \beta}, \tilde{\varphi}_{A}(\tilde{x})\right]=\left(\tilde{x}^{\alpha} \tilde{\partial}^{\beta}-\tilde{x}^{\beta} \tilde{\partial}^{\alpha}\right) \tilde{\varphi}_{A}(\tilde{x})+\left(\sigma_{A}{ }^{B}\right)^{\alpha \beta} \tilde{\varphi}_{B}(\tilde{x}),
$$

where $\tilde{\varphi}_{A}(\tilde{x}) \equiv \varphi_{A}(x)$.

In the above consideration, it has not yet been determined how many diagonal elements of $\xi_{a b}$ are -1 . In what follows, we show that $\xi_{a b}$ must be equal to the Minkowski metric $\eta_{a b}$. Here we note that $\operatorname{diag}\left(\eta_{a b}\right)= \pm(1,-1,-1,-1)$, that is, the overall sign is nothing more than the convention; what is physically important is the fact that the time direction is one-dimensional.

PNote that this prediction cannot be made by any other theory of quantum gravity. The observational fact that the gravitational force is of extremely long range should be regarded as the manifestation of the spontaneous breakdown of the $G L(4)$ invariance at the fundamental level. 
The invariant distance under the symmetry defined by $\tilde{M}^{\alpha \beta}$ is

$$
s \equiv \xi_{\alpha \beta}\left(\tilde{x}^{\alpha}-\tilde{y}^{\alpha}\right)\left(\tilde{x}^{\beta}-\tilde{y}^{\beta}\right) .
$$

If $\xi_{\alpha \beta} \neq \eta_{\alpha \beta}$, then there exists at least one extra dimension having the same metric signature as that of the ur-time (defined by the metric signature of $x^{0}=v_{\alpha}^{0} \tilde{x}^{\alpha}$ ). It is, therefore, possible to bring an arbitray spacetime point $\tilde{x}^{\alpha}-\tilde{y}^{\alpha}$ onto the hyperplane $x^{0}-y^{0}=0$ by a linear transformation (rotation) which leaves $s$ invariant.

For two arbitrary canonical fields $\Phi(x)$ and $\Phi^{\prime}(x)$, which need not be distinct, the canonical commutation relation between them at the equal ur-time is

$$
\left[\Phi(x), \Phi^{\prime}(y)\right]_{\mp}=0 \quad \text { for } x^{0}-y^{0}=0 .
$$

Since the symmetry which leaves $s$ invariant is not spontaneously broken, the vacuum expectation value of the left-hand side of (3.10) is invariant under the transformations of this symmetry. Rewriting it in terms of the tilde quantities, therefore, we have

$$
\left\langle 0\left|\left[\tilde{\Phi}(\tilde{x}), \tilde{\Phi}^{\prime}(\tilde{y})\right]_{\mp}\right| 0\right\rangle=0
$$

for any value of $\tilde{x}^{\alpha}-\tilde{y}^{\alpha}$.

According to the energy-positivity condition, (3.11) implies that an analytic function holomorphic (and vanishing asymptotically) in the lower-half plane and the one holomorphic (and vanishing asymptotically) in the upper-half plane have a common boundary value on the real axis. Hence Liouville's theorem on the entire function implies

$$
\left\langle 0\left|\tilde{\Phi}(\tilde{x}) \tilde{\Phi}^{\prime}(\tilde{y})\right| 0\right\rangle=0 .
$$

Thus all 2-point truncated Wightman functions for canonical fields vanish identically, that is, we have a trivial theory. ${ }^{\mathrm{q}}$ In order to have a nontrivial theory, therefore, we must set $\xi_{\alpha \beta}=\eta_{\alpha \beta}$.

We call the set $\left\{\tilde{x}^{0}, \tilde{x}^{1}, \tilde{x}^{2}, \tilde{x}^{3}\right\}$ the "physical spacetime"; this is, of course, the same thing as the ur-spacetime, though now it acquires not only metric structure but also the usual properties as a manifold. Then, as is seen from (3.8) with $\xi_{\alpha \beta}=\eta_{\alpha \beta}$, $\tilde{M}^{\alpha \beta}$ 's are the generators of the Lorentz transformations in the physical spacetime; they, of course, satisfy the commutation relations of the Lorentz algebra, as is easily confirmed from (3.5) by using the commutation relations for $\hat{M}^{\mu}{ }_{\nu}$ and those for $M^{a b}$ together with their commutativity. The ten generators $P_{\mu}$ and $\tilde{M}^{\alpha \beta}$ consititute the Poincaré algebra. In this way, the Lorentz invariance is derived as a consequence of

qFor the Dirac or Weyl theory, in which the Lagrangian density is linear with respect to the ur-time derivative of the field, we must consider also the canonical commutation relation $[\Pi(x), \Phi(y)]=$ $-i \prod_{k=1}^{3} \delta\left(x^{k}-y^{k}\right)$ for $x^{0}=y^{0}$, where $\Pi(x)$ denotes the canoninal conjugate of $\Phi(x)$. By the same reasoning as presented here, we have $\langle 0|\tilde{\Pi}(\tilde{x}) \tilde{\Phi}(\tilde{y})| 0\rangle=0$ except for the origin of $\tilde{x}^{\alpha}-\tilde{y}^{\alpha}$. But it is impossible to construct the corresponding 2-point truncated Wightman function such that it is a boundary value of an analytic function and invariant under the transformations which leave $s$ invariant. 
the spontaneous breakdown of the $G L(4)$ invariance. Thus, it is important to note that the Lorentz invariance is not a primary symmetry but a secondary one. ${ }^{\mathrm{r}}$

Finally, we remark that the results obtained above is not fully equivalent to the proposition that the action integral constituted by the elementary-particle fields only is Lorentz invariant. In order to obtain this proposition, it is further necessary that the relation $\left\langle 0\left|h^{\mu}{ }_{a}(x)\right| 0\right\rangle=v^{\mu}{ }_{a}$ holds. If it does, then replacing the veirbein field by its vacuum expectation value in $S_{M}$, we obtain the manifestly Lorentz-covariant action for the theory of elementary particles. Thus, in general, the manifestly covariant action is an approximation in which the gravitational effect on elementary particles is neglected.

\section{Spontaneous breakdown of translational invariance}

The translational invariance is, in a sense, a necessary condition for a physical law to be a physical law. Nevertheless, it is interesting to consider the possible consequences of the spontaneous breakdown of translational invariance. In this case, $\left\langle 0\left|g_{\mu \nu}(x)\right| 0\right\rangle$ is no longer independent of $x^{\lambda}$. Hence it can be regarded as the metric tensor of the a posteriori background spacetime. We may suppose that in a sufficiently small region the translational invariance is approximately unbroken and the argument made in $\S 3$ remains valid.

If we consider the $\kappa=0$ case, we can show that the 4-dimensional commutator between the veirbein fields vanish, and therefore (2.2) holds. Then for an arbitray function $f$, we have ${ }^{8}$

$$
\left\langle 0\left|f\left(g_{\mu \nu}{ }^{(0)}(x)\right)\right| 0\right\rangle=f\left(\left\langle 0\left|g_{\mu \nu}{ }^{(0)}(x)\right| 0\right\rangle\right)
$$

by extending the reasoning presented in deriving (3.11). Therefore, the background spacetime metric tensor, $\left\langle 0\left|g_{\mu \nu}{ }^{(0)}(x)\right| 0\right\rangle$, satisfies the classical vacuum Einstein equation (The contributions from the ghost fields, $b_{\rho}^{(0)}(x)$, etc., also vanish.). Thus, general relativity without the energy-momentum tensor $T_{\mu \nu}$ may be regarded as a consequence of the spontaneous breakdown of the translational invariance.

For $\kappa \neq 0$, of course, such a simple reproduction of the classical Einstein equation becomes impossible. Indeed, the covariant, mixed and contravariant components of the quantum Einstein equation may have a different vacuum expectation value.

\section{Summary and remarks}

In the present paper, we have shown how the Lorentz invariance of the elementaryparticle theory is derived on the basis of quantum gravity without assuming any concepts of classical physics a priori. It should be emphasized that the Lorentz invariance holds even if quantum gravitational interaction is present, as long as the translational invariance is not spontaneously broken.

${ }^{\mathrm{r}}$ This fact implies that the SUSY (supersymmetric extension of Poincaré invariance) cannot be a Lagrangian symmetry. 
At the operator level, we start with the ur-spacetime, which is an affine space having no metric structure. Our reasoning is heavily based on the BRS-formulated canonical operator formalism of the quantum Einstein gravity. In contrast with the classical Einstein gravity, it is independent of the pseudo-Riemannian structure; the general covariance is replaced by a quantum symmetry called the localtranslation BRS invariance. Furthermore, the local Lorentz invariance is replaced by the internal-symmetry BRS invariance. The internal-space metric $\xi_{a b}$ is unknown a priori; the concept of the tangent space must be forgotten because the base space has no structure as a manifold. The use of canonical formalism implies the assumption that the ur-time is one-dimensional. ${ }^{\mathrm{s}}$

At the representation level, the arrow of the ur-time is represented by the energypositivity condition for the Wightman functions. At the same time, the ur-spacetime is transmuted into the physical spacetime. The translational invariance is assumed not to be spontaneously broken; then both general linear invariance in the urspacetime and $\xi_{a b}$-rotational invariance in the internal space are spontaneously broken, but there remains an unbroken symmetry, that is nothing but the Lorentz invariance in the physical spacetime. Therefore, the Lorentz invariance cannot be a primary symmetry. Note that this situation is quite analogous to the spontaneous breakdown in the electroweak theory: Both $S U(2)_{L}$ and $U(1)_{Y}$ are spontaneously broken, but there remains an unbroken symmetry $U(1)_{\mathrm{em}}$; therefore, the electromagnetic gauge invariance cannot be a primary symmetry.

If the translational invariance is spontaneously broken, we no longer have the Lorentz invariance. When $\kappa=0$, the vacuum expectation value of the quantum gravitational field satisfies the classical vacuum Einstein equation. Thus the pseudoRiemannian geometry of general relativity can be regarded as a consequence of the spontaneous breakdown of translational invariance.

Finally, we note that if we imagine that the existence of the beginning of the Universe is a consequence of the spontaneous breakdown of the translational invariance of the $x^{0}$ direction only, then the spontaneously unbroken spatial 3-dimensions constitute a Euclidean space, that is, we have a flat, homogeneous and isotropic space without encountering the problem of event horizon. Thus it may be possible to avoid introducing the artificial hypothesis of inflation.

Note added: As discussed above, in the framework of quantum Einstein gravity, the "equal-time" commutation relations are set up with respect to the ur-time but not with respect to the physical time. Then there may arise the following question: "How can we see the local commutativity of field operators in the conventional quantum field theory on the Minkowski spacetime?" In the elementary-particle theory, the locality (or microcausality) is regarded as a fundamental postulate; indeed,

\footnotetext{
sThere might arise the misunderstanding that we are taking account of the possibilities of zerodimensional and multi-dimensional temporal spaces by confusing the internal space with the tangent space of the spacetime. This is not the case.
} 
January 23, 2014 9:33 WSPC/INSTRUCTION FILEローレンツ不変性の 起源

in the axiomatic quantum field theory, it is adopted as one of the axioms. In the conventional canonical quantization, the local commutativity automatically follows if we assume that the Lorentz invariance holds at the operator level. The latter proposition is not, however, a logical consequence of Einstein's special relativity, as emphasized in the Introduction.

In the framework of quantum Einstein gravity, the locality at the operator level is not manifest. Although we can show the vanishing of the vacuum expectation values of the commutators for the spacelike separation by using the reasoning analogous to the one developed from (3.9) to (3.11), it is not possible to establish the local commutativity in the complete sense. In order to obtain the manifestly Lorentzcovariant action integral for the elementary-particle fields, we must have the relation $\left\langle 0\left|h^{\mu}{ }_{a}(x)\right| 0\right\rangle=v^{\mu}{ }_{a}$, as stated in the last paragraph of $\S 3$. This condition is satisfied if we neglect the gravitational interaction. Thus, we see that the locality in the elementary-particle theory holds in the approximation in which gravity is assumed to be decoupled.

\section{Acknowledgments}

The author would like to thank Professor T. Kawai for valuable comments.

\section{References}

1. N. Nakanishi, Intern. J. Mod. Phys. D 20, 253 (2011).

2. N. Nakanishi, Publications Res. Inst. Math. Sci. (Kyoto Univ.) 19, 1095 (1983).

3. N. Nakanishi and I. Ojima, Covariant Operator Formalism of Gauge Theories and Quantum Gravity (World Scientific, Singapore, 1990), Chap. 5.

4. N. Nakanishi, Gen. Rel. Grav. 27, 65 (1995).

5. N. Nakanishi, Prog. Theor. Phys. 63, 2078 (1980).

6. R. Yoshida, Asia Univ. J. Soc. Gen. Res. 20, 53 (2013).

7. N. Nakanishi, Prog. Theor. Phys. 62, 1101 (1979).

8. N. Nakanishi, Prog. Theor. Phys. 111, 301 (2004).

9. N. Nakanishi, Prog. Theor. Phys. 66, 1843 (1981).

10. N. Nakanishi and I. Ojima, Phys. Rev. Lett. 43, 91 (1979). 